\title{
Perfil sociolingüístico de Frontera Corozal, Ocosingo, Chiapas
}

\section{The sociolinguistic situation of Frontera Corozal, Ocosingo, Chiapas}

\author{
Juan Jesús Vázquez Álvarez \\ (D) https://orcid.org/0000-0003-3094-2579 \\ Universidad Nacional Autónoma de México \\ Centro de Investigaciones Multidisciplinarias sobre Chiapas y la Frontera Sur \\ juanvazquezalvarez@gmail.com
}

\section{Resumen:}

Se presenta el panorama sociolingüístico de Frontera Corozal, un poblado chol fundado a partir de la migración de mediados del siglo pasado. Los choles de este lugar están en contacto lingüístico con lenguas dominantes extranjeras como el inglés y, desde luego, con el español, por su cercanía con los sitios arqueológicos de Yaxchilán y Bonampak. También mantienen una convivencia cercana con hablantes de otras lenguas indígenas como el tseltal y el lacandón. A pesar de la experiencia lingüística multilingüe en la comunidad y el deseo generalizado de que los hijos aprendan español, en este estudio se destaca que la mayoría de los niños tienen como lengua materna el chol, mientras que otros lo aprenden como segunda lengua. El habla de la población infantil y de algunos adultos de la comunidad de estudio dan indicios de una neutralización de la variación dialectal que caracteriza los lugares de origen de los colonos (chol de Tila vs chol de Tumbalá).

Palabras clave: choles, migración, variación dialectal, actitudes lingüísticas, transmisión intergeneracional.

\section{Abstract:}

This article presents the sociolinguistic situation of Frontera Corozal, a Chol town founded due to migrations from several municipalities of Chiapas during the second half of the last century. The Chol people in Frontera Corozal have extensive contact with socially dominant foreign languages such as English and Spanish, due to the town's proximity to the archeological sites of Yaxchilan and Bonampak. They also maintain close ties with speakers of other indigenous languages such as Tseltal and Lacandon. In spite of the multilingual situation and the value placed on acquiring full mastery of Spanish, this study demonstrates that most children in the community are still acquiring Chol as a first or second language. Data from child and adult conversations show evidence of neutralization of the dialectal variation that distinguishes the two varieties of Chol (Tila Chol and Tumbala Chol) spoken by the original colonizers of Frontera Corozal.

Key words: Chol people, migration, language variation, linguistic attitude, intergenerational transmission. 


\section{Introducción}

n este artículo se destaca la historia de la fundación de Frontera Corozal
y se presenta el panorama sociolingüístico del chol que se habla en esta
comunidad fronteriza entre México y Guatemala, incluyendo el contacto, la acomodación y las actitudes lingüísticas, así como los ámbitos de uso de la lengua local. En este sentido, es un estudio descriptivo desde la perspectiva de la sociología del lenguaje, con énfasis en el desplazamiento lingüístico y el bilingüismo (Wardhaugh, 1998 y Romero, 2017).

El chol, también denominado ch'ol y lakty’añ (López, 2005; Vázquez, 2011; Cruz, 2014), es una lengua maya de la rama tseltalana que tiene 251809 hablantes, según cifras del Instituto Nacional de Lenguas Indígenas (INALI, 2015a). Es una de las que cuentan con mayor cantidad de hablantes en Chiapas, después del tseltal y del tsotsil (INALI, 2015b; 2015c). ${ }^{1}$ Frontera Corozal es parte del municipio de Ocosingo cuya población indígena, según las estadísticas del Instituto Nacional de los Pueblos Indígenas, se divide en 174237 tseltales, 12364 choles, 1751 tsotsiles y 1129 lacandones (INPI, 2018). El acceso principal a Frontera Corozal desde Ocosingo es hacia la carretera que conduce a Palenque, Chiapas. Antes de llegar a dicha ciudad se toma el camino con rumbo a las zonas arqueológicas de Bonampak y Yaxchilán. En esta comunidad se encuentra el puerto de embarque en el río Usumacinta para acceder al último sitio arqueológico ya mencionado. Frontera Corozal quedó circunscrito dentro de la comunidad lacandona, como parte de los programas de reordenamiento poblacional del gobierno mexicano en las últimas décadas del siglo pasado. Por lo tanto, comparte una gran extensión territorial con poblaciones lacandonas y tseltales de Nueva Palestina. Gran parte de esta comunidad tiene bajo su cuidado la Reserva Integral de la Biosfera Montes Azules; por lo tanto, las reservas cercanas al núcleo poblacional no pueden ser destinadas para la siembra de productos agrícolas ni para introducir ganado vacuno, ni para practicar la cacería o la explotación maderera. Por esta razón, muchos habitantes de este lugar se dedican a actividades relacionadas con la atención del turismo nacional e internacional que visita las zonas arqueológicas o para practicar el ecoturismo. Ven la migración a centros urbanos como una opción en la búsqueda de empleos con una remuneración más sólida. A pesar de que el idioma chol sigue siendo el principal medio de comunicación entre los pobladores de Frontera Corozal, el español se usa para

\footnotetext{
1 En la misma fuente se reporta una población tseltal de 556720 y tsotsil de 487898 individuos.
} 
atender a los visitantes, incluso a los extranjeros. No se conocen personas de la comunidad que tengan un pleno dominio del inglés y que lo usen con los turistas, aunque se menciona el interés de algunos jóvenes por aprender otros idiomas (particularmente el inglés), para comunicarse con los visitantes sin necesidad de intermediarios. Cabe destacar que en los restaurantes o los embarcaderos se escucha a los guías foráneos haciendo traducciones del español al inglés, francés o alemán. Esta situación conlleva ciertas actitudes lingüísticas que pueden afectar la vitalidad del chol en la comunidad de estudio.

Los datos que se presentan en este artículo se obtuvieron a partir de la observación de las interacciones cotidianas en seis familias, la aplicación de entrevistas abiertas y el registro de narraciones tradicionales en audio o video. Fueron consultados maestros de una escuela primaria bilingüe, en la que además se me permitió presenciar la interacción comunicativa de los niños dentro y fuera del salón de clases. La población estudiada se agrupó en tres generaciones: los fundadores (abuelos), los hijos de estos (padres) y los nietos; referidos también como primera, segunda y tercera generación, respectivamente. En un primer momento trabajé con choles que son originarios y viven en Tila y Tumbalá. Estas personas evaluaron muestras de habla de la variante ajena con la finalidad de obtener una opinión al respecto. Los choles que participaron en este ensayo reconocieron la existencia de las dos variantes dialectales mencionadas en los estudios lingüísticos previos, lo que permitió comprender las actitudes lingüísticas con respecto a ambas. En un segundo momento, las mismas personas escucharon fragmentos de habla provenientes de Frontera Corozal. Con este ejercicio se logró reconocer que el chol que se habla en el poblado de nuestro interés está combinando propiedades léxicas y gramaticales de ambas variantes dialectales, opinión que también comparten los choles de la comunidad de estudio.

El artículo, luego de una introducción general, incorpora un esbozo histórico de la fundación del poblado. Se presenta el patrón de asentamiento de las familias choles y una sección sobre la presencia del turismo en el lugar para entender la vitalidad del chol en la demarcación. Posteriormente se detalla el uso de dicha lengua en diferentes ámbitos comunicativos. Fue necesario mencionar las principales características que se destacan en la variación dialectal del chol para dar razón, en el siguiente apartado, de los cambios que están ocurriendo en el chol de Frontera Corozal, puesto que se sugiere por parte de los hablantes un proceso de acomodación lingüística con predominancia de la variante dialectal de Tumbalá. En otro apartado, se ilustra el uso de esta lengua maya en algu- 
nos dominios. Por último, se destacan las principales actitudes lingüísticas de los choles frente a las dos variantes dialectales que coexisten en la comunidad y sus opiniones respecto al español u otra lengua dominante, como el inglés.

\section{La fundación de Frontera Corozal}

Según el historiador de la Selva Lacandona, Jan de Vos (1994), en 1821 dicha área selvática aún hacía honor a su nombre de despoblado o desierto de El Lacandón, por carecer de asentamientos humanos. Aunque cabe destacar que durante los siglos XVI y XVII, esta región estaba bajo el control de grupos cholanos, como fue el caso del poblado Dolores Sak Bahlan (Robertson et. al., 2010:3-4). El despoblado o desierto abarcaba los márgenes del río Usumacinta, donde actualmente se encuentra ubicado Frontera Corozal, municipio de Ocosingo Chiapas. ${ }^{2}$ Los datos derivados de reconstrucciones lingüísticas sugieren que la actual zona de El Lacandón estaba ocupada por grupos cholanos desde el año 250 d. C. Sin embargo, el poblamiento por parte de los choles que habitan en Frontera Corozal actualmente es producto de la migración de mediados del siglo pasado.

Según el historiador de El Lacandón, algo que cambió la condición de despoblado o desierto de esta zona selvática fue el experimento que llevó a cabo el tabasqueño Felipe Marín en 1859, cuando echó a las aguas del río Lacantún varias trozas de madera preciosa, mismas que recuperó, la mayoría de ellas, en la rivera de Tenosique, Tabasco. Este hecho despertó el interés de varias personas del mismo estado que estaban dispuestas a sacar provecho económico con la extracción y exportación del cedro y la caoba de El Lacandón. Por esta razón se indica que para 1870, varios tabasqueños establecieron pequeñas monterías en las orillas de los ríos Lacantún, Pasión y Usumacinta. El resultado es que, a mediados de dicha década, había 15 empresas que al año cortaban en promedio 200 trozas de madera preciosa cada una (De Vos, 2004:340). Puede considerarse que fue poco el daño que sufrió el bosque en ese tiempo si se compara con el deterioro que se le ocasionó un siglo después.

2 Este mismo autor ofrece una extensión aproximada de la Selva Lacandona de 15300 km²; constituida por 1. Las llanuras y declives del Golfo de México (que incluye el Alto Usumacinta y el Lacantún, así como la zona de Marqués de Comillas y Romano Sur) y 2. Sierra de los Lacandones (incluye el Nudo Diamante, las Sierras de la Colmena y del Caribe, la Meseta de Agua Escondida, las zonas de la Compañía Agua Azul y Romano Norte, el Valle de Ocosingo, las cuencas de los ríos Lacanjá, Azul, Perlas, Jataté, Tzaconejá, Dolores y Santo Domingo (De Vos, 1980:29). 
Una década antes de la culminación del siglo XIX (de 1897-1900), el gobierno federal puso en arrendamiento la parte del despoblado en donde se localiza actualmente Frontera Corozal en beneficio de la Casa Valenzuela, para la explotación de caoba y cedro, así como para la extracción de gomas y resinas (De Vos, 1994). Más tarde, en 1949, después de más de 70 años de extracción y exportación de maderas preciosas en rollo, el gobierno mexicano decidió cancelar el contrato (De Vos, 2004:345). Por eso, de 1949 a 1954, El Lacandón volvió a ser tierra de nadie, con presencia únicamente de pocos centenares de lugareños. Sin embargo, el gobierno de Miguel Alemán (1946-1952) promovió entre los campesinos sin tierra la ocupación de terrenos nacionales vírgenes, y aumentó de este modo la figura de las pequeñas propiedades, aunque también dejó abierta la posibilidad a la creación de pequeños latifundios a partir de terrenos nacionales, con el fin de incorporarlos a la producción agrícola o ganadera.

Es así como inicia el éxodo de choles y tseltales de distintos municipios chiapanecos que buscaban un lugar para establecer nuevos núcleos de población y con suficientes espacios para cultivar maíz y frijol, y continuó hasta las últimas décadas del siglo pasado con «jornaleros y avecindados temporales que vieron en la selva el edén perdido en donde podrían disfrutar de su vida más allá de la mera supervivencia» (González, 2004:429). En el mapa 1 se pueden apreciar las corrientes de población hacia El Lacandón, a principio de la década de 1960, desde Palenque, Ocosingo y Tenosique (De Vos, 2004:350). Muchos pobladores de Frontera Corozal y de comunidades vecinas recuerdan las penurias que pasaron por varios lugares de El Lacandón antes de establecerse definitivamente en dicho poblado fronterizo; algunos de ellos arribaron directamente a este lugar por invitación de familiares o vecinos en sus municipios de origen. 


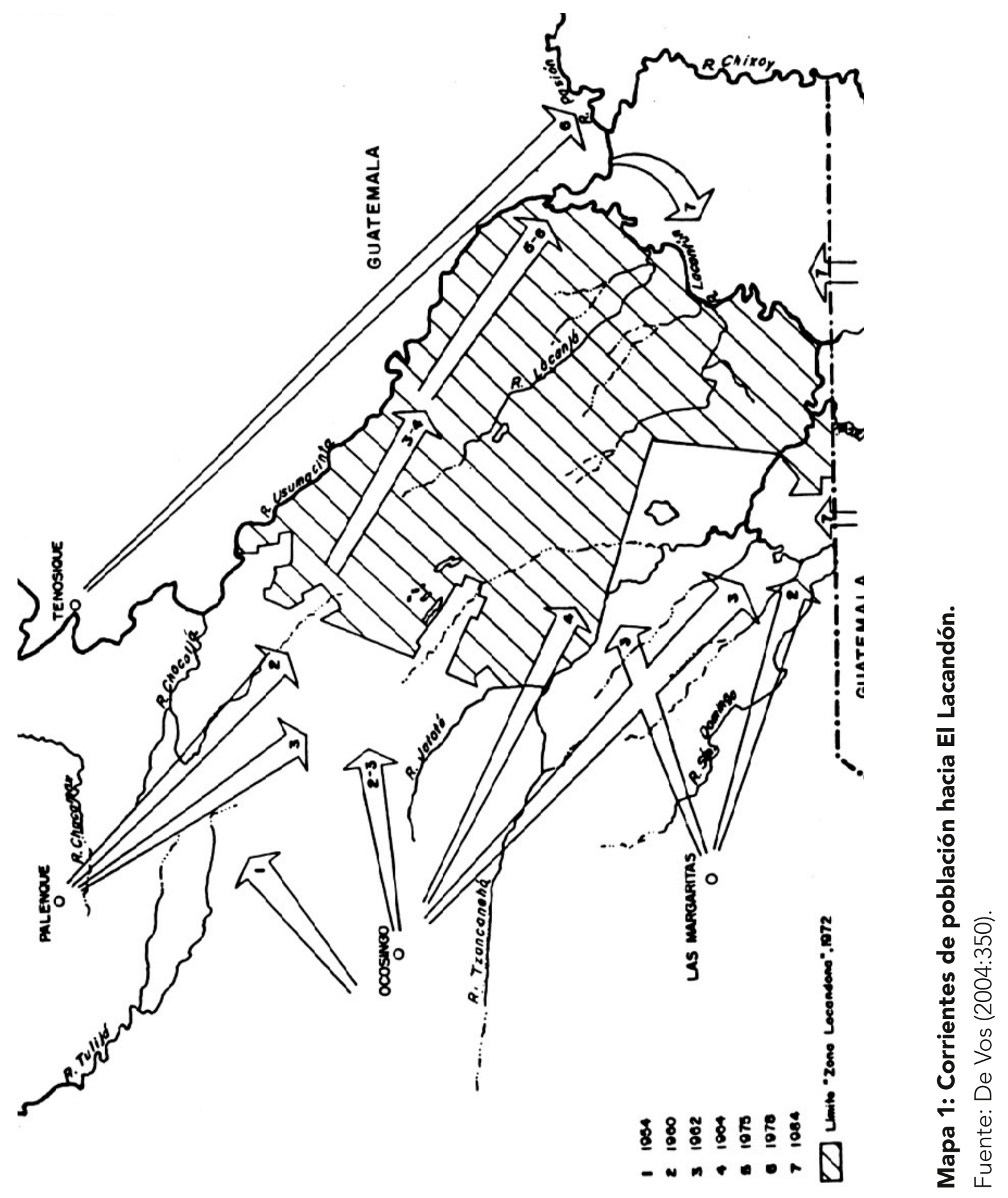




\section{Patrón de asentamiento familiar}

Los hogares típicos en Frontera Corozal se organizan en espacios amplios, los cuales pueden contener hasta cuatro casas que dan cobijo a la misma cantidad de familias nucleares. Estos «solares», Ilamados así por los lugareños, alojan a los fundadores de la comunidad, es decir, los abuelos, que pueden también estar rodeados de sus hijos y nietos, con quienes conviven la mayor parte del tiempo. Cuando una pareja contrae matrimonio, la casa se construye en los terrenos de la familia del esposo. De igual modo, aunque no es una práctica común, se permite que el esposo se establezca en los terrenos de la familia de la esposa, aun sin ser oriundo de la comunidad. Esta práctica contrasta con las restricciones que se observan en los lugares de origen de los colonos, donde no se permite que la mujer se quede a vivir en su comunidad con el esposo que no es del mismo lugar, debido al limitado acceso a las tierras destinadas al cultivo de productos agrícolas.

La mayoría de las casas tienen una forma rectangular, generalmente con una o dos divisiones, dependiendo de la cantidad de personas que la habiten permanentemente. Al lado de dicha construcción está la cocina, con dimensiones más pequeñas, en donde algunos miembros de la familia pasan mucho tiempo, especialmente las involucradas en la elaboración de alimentos, y los pequeños.

Es también común observar corrales cercados cuidadosamente con malla de alambre habitados por distintas aves. Para aquellas que tienen crías se les acondicionan espacios con cajas de madera y techos para protegerlas de las lluvias. Además del gallinero, algunas familias poseen chiqueros, ${ }^{3}$ otras siembran hierbas aromáticas como cebollín, perejil, una variedad de tomates pequeños, chiles y albahacas. Las abuelas y mamás de los pequeños pasan mucho tiempo en estos espacios al cuidado de los animales domésticos y las plantas. Ahí las abuelas usan el idioma chol con sus hijas; sin embargo, las hijas o nueras usan predominantemente el español como lengua de instrucción a sus respectivos hijos. Es importante señalar que las niñas suelen ser las encargadas de alimentar a las aves y a los puercos y de sacar o encerrar a los pollitos pequeños, y son también las responsables de traer las hierbas aromáticas para la cocina. Las interacciones comunicativas en los contextos descritos acontecen tanto en chol como en español. Cabe aclarar que estos hallazgos se basaron principalmente en observaciones directas hechas por el autor, con el apoyo de guías de entrevistas aplicadas en forma de conversaciones en la lengua chol con seis familias. Sin embargo, la

\footnotetext{
3 Pequeña cerca con techo para la crianza de marranos.
} 
tendencia observada aquí es la misma que se reporta en los estudios en la sociolingüística maya, ya que los padres socializan con sus hijos en la lengua española, mientras que la lengua local la aprenden de los abuelos o de otros parientes adultos (Romero, 2017).

También es necesario destacar que hay muchas familias de la segunda generación que han migrado a otros pueblos y ciudades en Chiapas, Campeche, Yucatán y Quintana Roo, y que regresan a Frontera Corozal por periodos cortos durante las vacaciones. En esos casos tienen ocasión de emplear la lengua local entre la primera y segunda generación y donde se nota la ruptura del uso del idioma con los de la tercera. La búsqueda de oportunidades laborales en las ciudades, el desplazamiento del lugar de origen por conflictos familiares o el matrimonio de parejas con distintas lenguas mayas o zoque son factores que inciden en el abandono del uso del idioma chol en la familia. El ejemplo de Juan, chol de 25 años (entrevista, 20/03/2018), sirve para ilustrar uno de estos hechos. Esta persona originaria de Frontera Corozal se casó con una lacandona con quien ha formado una familia con dos hijos. Actualmente vive en San Javier, una comunidad lacandona. Él se comunica en español con su esposa y sus hijos; incluso con su suegra, quien solo habla lacandón. Manifiesta que a él se le dificulta entender el lacandón, por lo que considera que no lo aprenderá; mientras que, por el contrario, su mujer tiene habilidades para aprender el chol. Juan piensa que no será necesario usar su lengua materna con su familia, por lo tanto, anticipa que sus hijos ya no aprenderán plenamente el chol o el lacandón, aunque, como él lo sugiere, sería bonito que fueran trilingües.

Ante lo expuesto, podemos decir que el patrón de asentamiento chol y las familias nucleares conformadas por hablantes de la lengua han contribuido a mantener parcialmente vigente su uso en la localidad. Los habitantes que conforman la primera generación juegan un papel central en el mantenimiento del chol en este lugar. El repertorio lingüístico de aquellos que representan la tercera generación determinará el destino de la vitalidad de esta lengua.

\section{Trabajo, turismo nacional e internacional}

La cuestión laboral de los habitantes de Frontera Corozal se limita en gran medida a las actividades asociadas a la agricultura y a la ganadería. Puesto que la vocación que se trajo de los lugares de origen era todo lo asociado con el cultivo 
de la milpa para el consumo familiar o el cultivo del café para fines comerciales, el trabajo en los nuevos centros de población consistió en «convertir el monte en milpas y potreros, y para conseguirlo empleaban un método sencillo, aunque laborioso: la roza-tumba-quema» (De Vos, 2004:348). Debido a la poca altura en la que se localiza el poblado y por el clima cálido, no fue posible introducir el cultivo del café, excepto en algunas zonas de Nueva Palestina, ocupado por tseltales, aunque tampoco tuvo éxito porque las plantas perecían en pocos años. Para obtener algo de ingreso económico, algunos pobladores choles se dedicaron a recolectar xate (Chamaedorea elegans), especie de palma ornamental de clima tropical, para venderla a los xateros o compradores que llegaban frecuentemente a la comunidad. También exploraron otras alternativas, como el cultivo del chile jalapeño. Sin embargo, requería muchos cuidados y algo de inversión por el uso de agroquímicos para lograr una buena cosecha. Con los ingresos económicos que se obtenían de estas actividades se podía comprar principalmente maíz y frijol que ofertaban vendedores guatemaltecos. Por otra parte, algunos colonos comenzaron a introducir el ganado vacuno, debido a que la gran mayoría poseía suficiente terreno para criarlo en potreros. Esta actividad ha tenido bastante éxito y por lo tanto muchos colonos poseen algunas cabezas de res; incluso, aquellos que no tienen, también han acondicionado sus terrenos como potreros y los rentan durante algunos meses del año. Esta actividad ha generado un empobrecimiento de los minerales de la tierra, situación que tiene un impacto en el crecimiento y engorda del ganado, pero sus dueños han buscado los mecanismos para compensar esta carencia comprando suplementos alimenticios, a pesar de que eso implica una inversión adicional. Puesto que la práctica de la ganadería se considera como una actividad rentable en la región, muchas personas están reduciendo sus espacios de siembra tradicional de maíz y frijol.

Con el tiempo, se ha dado un salto al cultivo de la palma de aceite. Don Juan manifiesta que, especialmente en la zona de Marqués de Comillas, han notado que la pastura es también pobre en minerales y por lo tanto no otorga suficientes nutrientes al ganado, y que para evitar gastos excesivos han optado por la siembra del hule o de la palma de aceite ya que, organizados, obtienen apoyo oficial mediante diversos incentivos económicos (entrevista, 15/08/2018). De acuerdo con Castellanos (2018), en Chiapas hay políticas gubernamentales destinadas a incentivar el cultivo de la palma de aceite, especialmente a pequeños productores, particularidad que va a distinguir a los colonos de la Lacandona y zonas aledañas. En estos poblados, un ejidatario puede disponer hasta de 50 hectáreas 
de terreno. En una encuesta aplicada a productores de palma de aceite a comunidades aledañas a El Lacandón, dicho autor encontró que un productor con 17.4 hectáreas destina 7.4 a la siembra de la palma de aceite y uno con 27.4 hectáreas puede destinar 10.3 hectáreas para el mismo fin (Castellanos, 2018:12).

Es incuestionable que la frontera sur de Chiapas está sufriendo una transformación en las formas tradicionales del uso de la tierra. Lo que se puede percibir es que en la mayoría de estas dinámicas relacionadas con las cuestiones agrarias está presente el chol como la principal lengua de comunicación, situación que ha permitido su mantenimiento y reproducción. Además, la noción de lo chol como grupo étnico ha permitido organizarse como grupo para gestionar algunos beneficios para la comunidad, como son servicios básicos o financiamiento de proyectos productivos. Cabe aclarar que en esta forma de organización se incluye a una minoría no hablante, debido a que también poseen idénticas formas de tenencia de la tierra.

Es importante puntualizar también que los hijos de los colonos están explorando otras alternativas laborales, en las cuales el uso del chol no siempre es posible. Por ejemplo, los que han encontrado trabajo como maestros bilingües, tanto en preescolar como en primaria, algunos se encuentran laborando en distintas escuelas de la región en donde no se habla necesariamente el chol, razón por la cual usan el español como principal medio de comunicación. ${ }^{4}$ Aun con población estudiantil mayoritariamente chol, como es el caso de la escuela primaria Jaime Torres Bodet y Javier Mina (ubicadas en Frontera Corozal), en donde todos los maestros son originarios de dicho poblado y por lo tanto todos hablan el chol, usan el español como lengua de instrucción y también para comunicarse con sus colegas. Cabe destacar que este sector de la población chol de Frontera Corozal está usando el español como la principal lengua de comunicación tanto en el ámbito familiar como en el escolar.

En este poblado se han generado otras opciones laborales por estar cerca de Yaxchilán, un famoso sitio arqueológico del periodo clásico maya. Año con año, especialmente en periodos vacacionales, hay presencia de visitantes locales, nacionales e internacionales, a quienes hay que ofrecer servicios de transporte, alimentación, hospedaje o guía al sitio arqueológico. Además, se requieren

4 En Chiapas, los servicios educativos federalizados incluyen el sistema educativo indígena bilingüe y el sistema monolingüe (español). En el sistema bilingüe se contempla la atención a niños hablantes de lenguas indígenas, donde se espera que la lengua de instrucción sea la materna del niño. Por el contrario, en el sistema monolingüe, la instrucción escolar acontece solamente en español. 
trabajadores para la custodia y el mantenimiento, mismos que están destinados para las personas "con estudio», como dice don Domingo, ya que se requiere el dominio de la lengua española (entrevista, 21/03/2018). La escolaridad a la que se refiere el entrevistado es el bachillerato, nivel escolar al que se tiene acceso en la comunidad a través del Colegio de Estudios Científicos y Tecnológicos del Estado de Chiapas (CECyTECH 33) y en los últimos años las licenciaturas que ofrece la Unidad Académica Crucero San Javier de la Universidad Tecnológica de la Selva (UTSelva).

Ante esta condición, algunos choles han conformado organizaciones o cooperativas de lanchas para transportar a los visitantes al sitio arqueológico o cruzar el río Usumacinta para visitar comunidades guatemaltecas, o viceversa. También existen organizaciones de taxistas que dan servicios locales o transportan viajeros a la carretera fronteriza que comunica con Palenque, Chiapas. Además, hay cooperativas que ofrecen servicios de hospedaje y alimentación para los visitantes. Estas últimas buscan obtener beneficios ofreciendo alimentos a los visitantes, ya que la gran mayoría de los turistas llegan temprano y se regresan por la tarde a Palenque, es decir, son pocos los que pernoctan en este lugar desde que pavimentaron la carretera. Los encargados de dichas cooperativas procuran capacitar constantemente a los trabajadores con el fin de ofrecer un buen servicio a los visitantes, especialmente en lo referente a la cocina. Respecto a la comunicación con los visitantes que no dominan el español, don Pascual, encargado del funcionamiento de la Cooperativa Nueva Alianza, considera que en los servicios del restaurante no ha sido necesario tener empleados que hablen inglés, ya que los grupos llegan regularmente acompañados de guías de turistas o de traductores. Sin embargo, manifiesta tener interés en capacitar a los jóvenes para que aprendan especialmente el inglés, con el fin de ofrecer un trato adecuado tanto en los servicios de hospedaje como de alimentación a las personas que llegan sin traductores (entrevista, 20/03/2018). En el contexto de la cooperativa, el turismo nacional y extranjero puede apreciar algunos elementos culturales que se muestran con orgullo, incluida la lengua chol.

Hace ya algunos años también se intentó conformar un museo comunitario en el que se exhibían algunas piezas de sitios arqueológicos cercanos no explorados aún, así como elementos de la cultura chol. Sin embargo, el proyecto no dio frutos y el lugar se encuentra actualmente abandonado. En todas las actividades relacionadas con el turismo predomina el uso del español, especialmente en lo que respecta a los servicios. Sin embargo, la comunicación en los espacios 
laborales, como los hoteles y los restaurantes, se realiza tanto en chol como en español.

\section{El idioma chol en Frontera Corozal}

Desde la reubicación de los colonos asentados en propiedad federal, como producto del Decreto Presidencial de 1972, los choles fundaron Frontera Echeverría y los tseltales Doctor Velasco Suárez, esto en honor a quienes eran en ese tiempo presidente de la República y gobernador del estado de Chiapas, respectivamente. Sin embargo, al paso del tiempo, decepcionados ante la falta del apoyo oficial prometido para la creación de los nuevos centros de población, decidieron cambiar esos nombres por los de Frontera Corozal y Nueva Palestina, respectivamente. ${ }^{5}$ Los mismos habitantes de Frontera Corozal reconocen que algunos choles se fueron a Palestina y que algunos tseltales Ilegaron a Frontera Corozal. En este último poblado se menciona la presencia de algunas familias tseltales; se destaca cómo los abuelos eran monolingües en tseltal; los hijos, bilingües con pleno dominio del tseltal y del chol, así como comprensión del español, mientras que los nietos llegaron a ser hablantes solamente del chol y, desde luego, del español. Don Domingo también menciona a una persona de la misma comunidad a quien pensó por mucho tiempo que era cholhablante, hasta que un día descubrió que esta lengua la aprendió en la edad adulta porque su lengua materna era el tseltal.

Las personas entrevistadas manifestaron que la gran mayoría de los habitantes de la comunidad hablan el chol con pocas excepciones. Estas últimas son mujeres que vienen de Guatemala o de algún otro lugar en donde no se habla esa lengua. Y aseguran que los hijos de estas personas aprenden el chol en las escuelas primarias en donde la gran mayoría de los niños lo usan en su interacción cotidiana, a pesar de que los maestros no lo usan en las aulas como lengua de instrucción.

Algunos choles reconocen que hay padres que están comunicándose en español con sus hijos en su interacción cotidiana. Estos niños tienen entonces como primera lengua el español, aunque obviamente están expuestos al chol en el contexto comunitario o en el hogar, con los abuelos. Es sin embargo en la escuela donde llegan a reforzar su competencia comunicativa en chol. El director

\footnotetext{
5 Se bautizó como Frontera Corozal en razón de que el poblado se asentó en los límites entre México y Guatemala y porque ahí abundaban plantas de palma de corozo.
} 
del plantel Jaime Torres Bodet manifiesta que hay niños que llegan a la primaria comunicándose solamente en español y que ese patrón se observa en primero y segundo grados. En tercero, estos niños aparentemente monolingües comienzan a interactuar en chol con sus compañeros de juego durante el recreo, a pesar de que en el hogar siguen usando el español. ${ }^{6} \mathrm{Al}$ preguntarle a los maestros respecto a la actitud que adoptan sobre esta situación, el director asegura que lejos de prohibirles, motivan a estos niños a que usen la lengua local y a los niños hablantes les piden que no se burlen de aquellos que están empezando a usar el chol. Es evidente que el uso de la lengua local en el contexto escolar no es parte de alguna planificación lingüística, sino producto del bilingüismo de muchos niños que asisten a esas escuelas. A pesar de que los hablantes de la segunda generación consideran bueno seguir usando la lengua materna, se nota que en la práctica se comunican en español en la socialización con sus hijos. En este sentido no hay indicios de una apreciación de los beneficios de un bilingüismo chol-español; por el contrario, el español es ya la primera lengua de muchos mayas (Romero, 2017).

\section{Variantes dialectales del chol}

Tal como apuntó De la Torre, el municipio de Ocosingo es un «territorio tan grande y ha sido el más despoblado hasta los años cuarenta; en la actualidad sigue dando cabida a cientos de campesinos de diversas lenguas indígenas y, por tanto, es el que presenta una mayor diversidad de población en cuanto a lenguas se refiere» (De la Torre, 1994:45). Esta observación se hizo antes de que parte del territorio de este municipio diera lugar a la formación de dos nuevos municipios chiapanecos, que son: Benemérito de las Américas y Marqués de Comillas. Sin embargo, la dinámica poblacional que observó De la Torre en los años ochenta sigue vigente de algún modo, especialmente para la zona de Marqués de Comillas. De Vos (2004: 355) decía que ya no hay tierra para repartir, pero hay un aumento demográfico debido a la alta tasa de natalidad. La movilización

6 Durante una visita a la escuela Jaime Torres Bodet se registró una breve conversación en chol con un niño de tercer grado de primaria, quien no mostró dificultades para responder a preguntas puntuales, pero ante el cuestionamiento sobre cuántos hermanos tiene, respondió: uxp'ej, dando a entender que tiene tres hermanos. En este ejemplo se nota que este niño aún no ha adquirido plenamente el uso del clasificador numeral para referente humano, que es tyikil; el uso del clasificador genérico p'ej en este contexto es inaceptable, y por esa razón su compañero de juego, que evidentemente tiene buen dominio del chol, lo corrige enfatizando que la respuesta apropiada debe ser uxtyikil. 
poblacional es una constante; por ejemplo, algunos colonos comúnmente venden sus tierras a compradores que pertenecen al grupo lingüístico predominante de la comunidad, tal como se puede observar en Frontera Corozal, Benito Juárez, Delicia y Loma Linda, que son poblaciones choles.

Los choles que han colonizado desde hace algunas décadas el territorio de El Lacandón provienen de todos los municipios con predominancia poblacional chol; es decir, Sabanilla, Tila, Tumbalá, Salto de Agua y Palenque. En algunos casos es posible inferir el lugar de origen de la mayoría de los pobladores de un nuevo centro de población con base en la toponimia, por ejemplo: Nuevo Tila, Nuevo Tumbalá y Nuevo Sabanilla son poblaciones choles; Nuevo Francisco León es un poblado con hablantes de zoque y Nuevo Tenejapa es un poblado tseltal (véase De Vos, 2002: 124-130). Pero además, a veces el dominio de cierta variante dialectal también es un factor a considerar, como sucedió en la distribución de dos barrios de Frontera Corozal, al nombrarlos: Barrio Nuevo Tila y Barrio Tumbalá, por ser el municipio de origen de la mayoría de las personas que ocuparon estas zonas del poblado, y por lo tanto, con una distribución dialectal bien definida. Para el caso chol, esto significa que hay un fuerte vínculo con el lugar de origen y por lo tanto pesa la identidad étnica, pero también juega un rol importante la variante del chol de los dos municipios más representativos (Tila y Tumbalá).

De Vos (2004:350), mostró por medio de un mapa que las corrientes de población por la zona norte de El Lacandón (que es donde se ha concentrado la población chol) iniciaron en 1960 y se prolongaron hasta mediados de la década de 1980 a la zona de Marqués de Comillas (véase mapa 1). En el trayecto confluyeron tanto hablantes del chol de Tila como del de Tumbalá, quienes usaron sus lenguas maternas como el idioma de comunicación cotidiana entre ellos. En estos contextos ya no cobró relevancia el problema de la inteligibilidad que es aún común escuchar en los municipios de origen.?

Es evidente que entre los choles de Frontera Corozal, así como en las comunidades choles aledañas, aún reconocen la existencia de las dos variantes dialectales de esta lengua maya: el chol de Tila y el chol de Tumbalá. Algunas personas entrevistadas en Frontera Corozal afirman que en este lugar se está favoreciendo el uso de la variante de Tumbalá, haciendo alusión al factor cuantitativo, es decir, esto se debe a que la mayoría de los colonos usan el chol de Tumbalá.

\footnotetext{
7 Appel y Muysken (1996) destacan las connotaciones sociales de las lenguas, que afloran cuando los hablantes asumen una identidad con características particulares con respecto a otro grupo. Por ejemplo, hay valoraciones afectivas entre los hablantes del chol de Tila frente a los de Tumbalá y viceversa, quienes respectivamente manifiestan con orgullo poseer "un buen chol" (uts'atybä).
} 
En términos del uso de una u otra variante, encontramos que los de la variedad tumbalteca no encuentran dificultades sociales en la interacción cotidiana, mientras que los que usan la variedad de Tila sí. Por ejemplo, don Juan, quien usa la variedad de Tumbalá por ser el idioma que le inculcaron en el hogar, manifiesta conocer palabras de la variedad de Tila, sin darle mucha importancia; afirma que la variedad que no es la suya es la que predomina en el barrio Nuevo Tila de Frontera Corozal (entrevista, 20/03/2018). Sin embargo, los que usan la variedad de Tila presentan ciertas dificultades en su interacción cotidiana por ser la variedad «marcada» o «señalada» por los usuarios de ambas variantes dialectales. Tenemos el caso de don Domingo (chol de Tila), quien reconoce que él y su familia se han adaptado a la forma de hablar de Tumbalá. Esta realidad es confirmada por su hijo, quien manifiesta hablar como los tumbaltecos (entrevista, 21/03/2018). Pero es el profesor Felipe, director de la primaria bilingüe Jaime Torres Bodet, quien da detalles sobre las actitudes de los niños con respecto al uso del chol de Tila y sus efectos lingüísticos.

La mayoría de acá de la comunidad son de variante Tumbalá y pocos eran de la variante Tila, Sabanilla. Había una gran diferencia. Y muchas veces, la lengua que ellos empleaban [variedad de Tila] se escuchaba muy chistoso para ellos [niños que usan la variedad de Tumbalá] y se burlaban. Eso nosotros no lo permitíamos, pero ya ve que uno no está pegado con los niños, de repente así se está jugando y ya se están burlando. Y cuando viene uno a ver ya están reportando: "maestro me está diciendo así, se está burlando". Y uno como maestro se pone a platicar con el niño, que no debe hacer eso, es una variante que ellos tienen. Así como también escuchan raro la lengua que hablamos, ellos no dicen nada, pero nosotros sí. Entonces los niños se medio controlan. Pero a raíz de eso, también se ha ido modificando su lenguaje, por ejemplo, se han ido acoplando a lo de Tumbalá. Entonces es muy raro escuchar orita con la variante de Tila. Hasta con los señores, como que ha ganado terreno el de Tumbalá, porque son mayoría. Entonces los señores que hablan de esa forma pues yo creo que también ya les ha dado pena: "quizás se van a burlar de mí", y se han ido acoplando. Los de Tila ya están hablando como los de Tumbalá. Cosa que para mí no debió ser así, allí no se puede cambiar. Como la mayoría de aquí es de lengua de Tumbalá, pues también la mayoría de los niños se han ido acoplando. Porque también han tenido ese miedo: "si lo digo, se va a reír, se va a burlar de mí". Es un poco complicado ir corrigiendo eso (entrevista, 21/03/2018).

Las opiniones del director de la escuela coinciden con los resultados de la investigación de De la Torre (1994), en el sentido de que se destacan numérica- 
mente los hablantes de la variante dialectal de Tumbalá y por lo tanto han impuesto dicha variedad a los choles que vienen de algún municipio en donde se habla la variedad de Tila. Este investigador realizó recorridos en varios centros de población de El Lacandón a mediados de la década de 1980 y en ese entonces, como ahora, encontró que:

Es predominante, en los pueblos donde se levantó la encuesta, la población proveniente del municipio de Tumbalá; lo cual no sólo nos permite entrever que éste es el municipio que provee, en mayor medida, de población a esta área de expansión, sino que también corresponde a la variante dialectal de mayor extensión en esta lengua; con lo cual se marca el predominio de ésta en el área de poblamiento. La población proveniente del municipio de Tila es menor que la de Tumbalá y su forma dialectal, por lo tanto, también es minoritaria. En este sentido, es posible pensar que algo sucederá en términos de asimilación o predominio en determinada variante... no es posible determinar si convivirán y se mantendrán diferentes o si se asimilarán y se generarán nuevas diferencias debido al intercambio y contacto con alguna otra lengua con la que convivan (De la Torre, 1994:84).

Efectivamente, se nota que en este poblado hay un fenómeno de asimilación con predominio hacia la forma dialectal que corresponde al chol de Tumbalá. Los propios hablantes del chol de Tila reconocen estar usando la variedad ajena a la suya y por su parte, los de la variedad de Tumbalá manifiestan que los de Tila se están acomodando a la forma de Tumbalá. Esta situación deja entrever la posibilidad de abrir nuevos senderos en los estudios sociolingüísticos del chol para ser explorados en contextos de asentamientos choles de reciente formación con la presencia de las distintas variantes dialectales de la lengua en cuestión. La observación informal permitió notar cambios en la fonología, morfología, sintaxis y, por su puesto, en el léxico.

Para poder abordar los posibles cambios que pudieran estar aconteciendo en el chol que se habla en Frontera Corozal es preciso destacar algunas características de la variación dialectal de esta lengua maya. En estudios lingüísticos previos, López (2005), Arcos (2009), Vázquez (2002; 2011), se reporta la existencia de dichas variantes dialectales. En tales estudios lingüísticos se han destacado variaciones en el nivel morfológico y léxico. En cuanto a la fonología, Vázquez (2011) menciona que algunos sonidos africados y fricativos tienen una realización retrofleja en Tumbalá, característica que no se escucha en Tila. También es innegable la existencia de diferencias sobresalientes en el patrón entonacional. 
Salvo estudios detallados sobre la fonología, se puede adelantar que la segunda generación de pobladores que recibieron input del chol de Tumbalá está abandonando la pronunciación retrofleja de algunas consonantes y está en proceso la unificación en las características prosódicas respecto a la variedad de Tila, lo cual estaría en consonancia con las tendencias hacia el cambio motivado por contacto (Matras, 2009). En el plano morfológico se destaca el empleo de diferentes morfemas para las marcas de aspecto perfectivo y progresivo, tal como se puede apreciar en el cuadro $1 .{ }^{8}$ Estos morfemas, de acuerdo con los choles, son los que dan indicios de la procedencia de los hablantes.

Cuadro 1: Formas de marcación aspectual en distintos dialectos del chol.

\begin{tabular}{|l|l|l|}
\hline Aspecto & Tumbalá & Tila \\
\hline Perfectivo & tsa' / ta' & tyi \\
\hline Progresivo & woli / wo & chonkol / chonko \\
\hline
\end{tabular}

Fuente: Vázquez, 2011:4-5.

En el nivel morfosintáctico, en Tumbalá se destaca el uso del clítico irreal ik en la negación y existe un pequeño grupo de modificadores adverbiales que no se escucha entre los choles de Tila (véase ejemplo 2, más adelante).

En lo que respecta a las diferencias léxicas, hay muchos ejemplos (véase cuadro 2). A continuación, se enlistan algunos.

Cuadro 2: Ejemplos de variación léxica.

\begin{tabular}{|l|l|l|}
\hline Español & Tumbalá & Tila \\
\hline Niño & ch'ityoñ & alob \\
\hline Niña & xch'ok & xk'aläl \\
\hline Piedra & xajlel & tyuñ \\
\hline Chayote & ñi'uk' & ch'ijch'um \\
\hline Flauta & amäy & jaläl \\
\hline Alacrán & siñañ & tya'chä' \\
\hline Araña & am & chiwoj \\
\hline
\end{tabular}

Fuente: Datos de campo.

\footnotetext{
${ }^{8}$ En el chol de Tila también se usa el perfectivo tsa' o ta' cuando el morfema aloja algún clítico de segunda posición; en caso contrario, se emplea la forma tyi (para más detalles véase Vázquez, 2002:97-98).
} 
Cabe destacar que, en consonancia con lo reportado en las investigaciones lingüísticas, los hablantes del chol reconocen la existencia de dos formas particulares de habla en esta lengua maya; es decir, todos los hablantes son capaces de notar formas de habla ajenas a la variedad propia.

En Frontera Corozal coexisten las dos variantes dialectales mencionadas, debido al origen de los primeros colonos de esta localidad, que provenían de municipios en los que se habla el chol de Tila o el chol de Tumbalá. A reserva de estudios fonológicos sistemáticos, en líneas anteriores se apuntaron algunos cambios en la fonología del chol que se habla en Frontera Corozal. En el contexto escolar, según el profesor Felipe Díaz, en la escuela Jaime Torres Bodet la mayoría de los niños choles dominan la variante de Tumbalá. Estos se mofan de la forma de hablar de los que usan la variedad de Tila. Por esta razón estos últimos han aprendido la forma de Tumbalá para su uso en el contexto escolar, mientras que en el hogar usan la variedad propia. Esto nos hace pensar que los niños que están aprendiendo o están reforzando el chol en el contexto escolar también están aprendiendo la variedad de Tumbalá.

\section{Cambios en el chol de Frontera Corozal}

Al preguntar sobre el uso de la lengua chol en Frontera Corozal y sobre la intuición de la existencia de dos variantes lingüísticas se destaca en las respuestas la palabra chol xajlubil, que significa 'revuelto', 'mezclado' o 'combinado'. Dicha expresión hace referencia a que los primeros colonos eran hablantes tanto del chol de Tila como del chol de Tumbalá; y se enfatiza además que esta última es la que ha predominado. Los propios ancianos oriundos de lugares donde se hablaba el chol de Tila reconocen que ellos y sus familias están cambiando para acercarse más a la variedad de Tumbalá. Ante tal panorama, consideré pertinente analizar algunas emisiones de habla obtenidas en contextos de uso natural, que permitieran entender las suposiciones de los propios hablantes. Algunos hallazgos se detallan a continuación.

En el plano fonológico, los choles (especialmente los de Tila) identifican como particularidad saliente de la forma de la variedad de Tumbalá un habla como con «eco»; en términos lingüísticos se trata de la pronunciación retrofleja de algu- 
nos sonidos. ${ }^{9}$ Don Manuel, un hablante de Tila, dice: xojokña muk'ob tyi ty'añ li $x k^{\prime} u k^{\prime}$ witsob (los de Tumbalá hablan de X manera, es decir, xojokña). ${ }^{10}$ Con base en las opiniones de algunos choles de Frontera Corozal y de mi competencia como hablante de este idioma puedo afirmar que, en efecto, en este lugar se está perdiendo esa propiedad de la fonología del chol de Tumbalá. Aunque está todavía presente en algunos ancianos fundadores de la comunidad, los hijos de estos ya no la conservan y mucho menos los hijos de los pobladores que pertenecen a la variedad de Tila. Es probable que el cambio de la pronunciación retrofleja a alveopalatal en algunas consonantes africadas y fricativas por parte de los choles de Tumbalá se deba en un primer momento a la convivencia con los choles de Tila y posteriormente por el contacto con el español, una lengua dominante que no tiene sonidos retroflejos. Hay que recordar que la lengua de comunicación en las asambleas para organizar la fundación de Frontera Corozal aconteció en chol, pero más tarde, con la llegada de las escuelas, la radio y la televisión, la lengua española se introdujo plenamente en la comunidad.

Otra propiedad saliente de la variante dialectal chol de Tumbalá es su patrón entonacional muy diferente a la variedad de Tila. Las investigaciones lingüísticas realizadas hasta ahora sobre el chol no abarcan el tema de la entonación. Por esta razón, en este estudio procuré opiniones tanto de cholhablantes de los municipios de Tila como de los de Tumbalá, con respecto a algunos materiales audio-grabados que provienen de algunos pobladores de Frontera Corozal, quienes manifestaron usar la variedad tumbalteca. Con este ejercicio se pudo constatar que la entonación que caracteriza la variedad tumbalteca ya no está fuertemente presente en el chol que se usa en Frontera Corozal, a pesar de que sus usuarios aseguran dominar dicha variedad. Pero esta particularidad trasciende a otros niveles de la gramática de esta lengua maya, tal como se puede constatar en los datos que destacan a continuación.

En líneas anteriores se mencionó que los hijos de los fundadores han aprendido la variante de Tumbalá, con las consideraciones señaladas en el aspecto de la fonología. También es necesario destacar que los cambios lingüísticos están ocurriendo del mismo modo en algunos ancianos que son originarios de Tila,

9 Es probable que esta propiedad saliente en la pronunciación de la $x$ y la ts entre los choles de Tumbalá sea un reflejo del contacto lingüístico de las Tierras Bajas mayas mencionadas por Law (2017). Cabe destacar que el q'anjob'al, una de las lenguas involucradas en dicho contacto, tiene los mismos sonidos retroflejos, pero aparecen en ambientes contrastivos con sus correspondientes sonidos alveopalatales (Zavala, 1992).

10 Se refiere a la imitación del eco en la pronunciación retrofleja de algunas consonantes en Tumbalá. 
como es el caso de don José, con una edad aproximada de 80 años. En el plano léxico, esta persona ha sustituido algunas palabras que son representativas de su variante dialectal por las de Tumbalá. Por ejemplo, nombra sistemáticamente ñi'uk' al 'chayote', xajlel a 'la piedra', ch'ityoñ a 'los niños' y xch'ok a 'las niñas'. El registro de tales emisiones aconteció cuando en el hogar de don José estaban de visita por unos días choles del municipio de Tila, quienes en la conversación, durante la comida, nombraban ch'ijch'um al 'chayote'; a pesar de ello don José usó la forma que ha adoptado de la variante de Tumbalá. Está también el caso de don Domingo, otra persona originaria del municipio de Tila, a quien se registró el uso sistemático de la palabra e'tyel para 'trabajo', en otro evento comunicativo. Cabe señalar que la forma característica para este significado en su municipio de origen es troñel. Las incorporaciones léxicas ejemplificadas aquí son las esperadas en la jerarquía de préstamos léxicos, ya que los sustantivos son los que se transfieren con mayor frecuencia (Field, 2002).

Más cambios en la forma de habla de don José también están presentes en el plano morfosintáctico. Por ejemplo, en el ordenamiento de los clíticos de segunda posición, el clítico aspectual tyo lo coloca al final de la frase o la palabra, por ejemplo:

\section{(1) Bakityo}

'hasta donde'

El orden en la variedad de Tila sería batyoki. En otros usos de los clíticos en la variante dialectal de Tumbalá, al morfema para indicar la negación se acompaña del clítico =ik; resultando en ma'añik; peculiaridad que se registró también en los usos de la persona oriunda de Tila, don José (2). ${ }^{11}$

(2) ma'añik ta' ñämi ja'al

NEG PFV pasar lluvia

'No pasó la lluvia'

Por otro lado, en la lengua chol se ha descrito la existencia de modificadores, algunos con funciones adverbiales (véase Vázquez, 2011:218-220). Hay modificadores que son exclusivos de la variante de Tumbalá, por ejemplo: tyo'o ('en

${ }^{11}$ Si empleara las formas de la variedad de origen de esta persona, tendríamos: ma'añ tyi ñämi ja'al. 
vano'), laj ('todo') y k'uñtye' ('despacio' o 'lentamente'); las respectivas formas para Tila son: Ioloñ, lu' y xuk'ul. Estos cambios fueron registrados en la forma de habla de don José, quien por ejemplo dijo:

(3) K'uñtye' mi i-ñäme karu

Despacio IPFV A3-pasar carro ${ }^{12}$

'El carro pasaba despacio'

Por último, tal como se ilustró en el cuadro 1, cada variante dialectal del chol tiene morfemas particulares para los aspectos perfectivo y progresivo. En las siguientes emisiones de don José (oriundo de Tila), se ve que ha sustituido las formas de su lugar de origen por las respectivas formas tumbaltecas: tsa' o ta' (para el perfectivo) y woli o wo (para el progresivo). ${ }^{13}$

(4) Ta' kä-le- $\varnothing \quad$ otyoty=bä

PFV quedar-STAT-B3 casa-REL

'Se quedó la casa (dejamos la casa)'

(5) Wo i-k'ajty-iñ- $\varnothing-o^{\prime}$

PROG A3-preguntar-TD-B3-PL

'Están preguntando (sobre algo)'

La sustitución de algunos elementos gramaticales en el habla de los choles de Frontera Corozal explica la idea común en las personas entrevistadas de que en este lugar ha predominado la variante del chol de Tumbalá; incluso los que son originarios de Tila y Sabanilla "ya hablan como tumbaltecos», o por lo menos hay un intento de acomodación a la variedad ajena. Por esta razón, las evidencias de la selección de algunos elementos léxicos y gramaticales registradas en la forma de habla de don José son muy ilustrativas, en el sentido de que confirman las opiniones que se obtuvieron de algunos choles de Frontera Corozal. Los datos presentados muestran que hay rasgos léxicos y gramaticales salientes en cada variante dialectal. Al cambiar el léxico, el orden de los clíticos, los modificadores verbales más comunes y un par de formas aspectuales, da la impresión de que

\footnotetext{
${ }^{12}$ Abreviaturas: A: Marcas de persona del juego A (ergativo), B: Marcas de persona del juego B (absolutivo), IPFV: Aspecto imperfectivo, PFV: Aspecto perfectivo, PL: Plural, PROG: Aspecto progresivo, REL: Clítico relativizador, STAT: Sufijo estativo, TD: Sufijo para verbo transitivo derivado.

${ }^{13}$ Véase nota 9, sobre el uso de los alomorfos tsa' o ta' del perfectivo en Tila.
} 
se ha abandonado la forma de habla de Tila, como en el caso de don José. Hay que destacar también que al parecer está en proceso un cambio en el patrón entonacional; don Manuel (hablante del chol de Tila) dice: «es chol de Tumbalá, pero es más parecido al chol que nosotros usamos», mientras que Jorge (chol de Tumbalá), manifiesta: «este chol ya no es cantado como nosotros lo hablamos».

El chol que usan los hijos de los primeros pobladores de Frontera Corozal (segunda generación), así como los nietos (tercera generación) está perfilando una variedad intermedia entre el chol de Tila y de Tumbalá, que se caracteriza por un cambio en el patrón entonacional y en la pérdida de la pronunciación retrofleja de algunos sonidos. La acomodación a la variedad tumbalteca que muestran algunos ancianos provenientes de Tila y Sabanilla, por otro lado, indica que desde la variedad tileca existe también una tendencia al cambio en el plano léxico y morfosintáctico. El cambio continúa de la mano del avance de las generaciones; seguirá por algún tiempo, puesto que como se detalla en el siguiente apartado, la lengua local aún está presente en diversos dominios de uso cotidiano.

\section{Dominios de uso de la lengua materna}

Para Sánchez-Marco, uno de los conceptos centrales en los estudios sociolingüísticos se refiere a «los dominios de uso». Tal concepto hace alusión a las condiciones en las cuales unas veces se emplea cierta variedad de una lengua dada, mientras que otras se modifica la variedad dialectal o incluso la misma lengua, según la competencia lingüística de los hablantes (Sánchez-Marco, 1976:150). En esta sección se presentan algunas informaciones de las interacciones lingüísticas cotidianas obtenidas a partir de la observación directa y de la aplicación de entrevistas. Los espacios que se toman en cuenta son principalmente el hogar, la escuela, la iglesia y aquellos en los cuales se desarrollan algunas actividades económicas como el comercio o la prestación de servicios turísticos a visitantes nacionales y extranjeros.

Aunque podría pensarse que cada ámbito o espacio de uso tiene una correlación directa con una lengua específica, lo que se observa en Frontera Corozal es algo complejo, pues en todos está presente también el español. Por ejemplo, en lo que respecta al hogar, los fundadores de la comunidad, es decir, los adultos mayores, usan el chol para comunicarse con la esposa y sus hijos; pero se nota que los hijos están comunicándose en español con sus respectivos hijos. 
Además, la gran mayoría de la generación joven, incluyendo nietos y bisnietos, han tenido una instrucción escolar en la cual ha predominado el español como lengua de enseñanza y en la que los libros de texto que se usan están escritos también en español. Algunos fundadores de la comunidad manifestaron en las entrevistas que buscaron inculcar la lengua española a sus hijos, situación que está siendo reproducida en los niños, pero muestran sorpresa de que las nuevas generaciones estén hablando o por lo menos entienden el chol. La explicación que los padres ofrecen para esta situación lingüística es que lo aprenden al escuchar conversaciones en chol de los adultos en la casa; cabe señalar que muchos niños viven en casas contiguas a las de los abuelos y por esta razón están expuestos constantemente al repertorio lingüístico de los adultos mayores. Otra explicación nos la ofrece el director de la escuela primaria bilingüe Jaime Torres Bodet, profesor Felipe, quien afirma que muchos niños del primer ciclo (primero y segundo grado) usan el español para su comunicación porque muchos de ellos lo aprendieron como su primera lengua. Sin embargo, para el último ciclo (quinto y sexto grado), ya hablan el chol como producto de su interacción con sus compañeros de clases o de juego durante el recreo. ${ }^{14} \mathrm{El}$ director de dicha escuela primaria ve esta situación como algo positivo para el mantenimiento del chol, por eso organizan pláticas para motivar a los niños a valorar la lengua local.

En esta demarcación, dos escuelas primarias pertenecen al sistema de educación indígena bilingüe, en el que, por norma, el proceso de enseñanza-aprendizaje debe desarrollarse principalmente en lengua indígena. Sin embargo, en estos ámbitos la lengua que se utiliza es el español, aunque algunos maestros reconocen usar esporádicamente el chol para dar instrucciones en las tareas escolares. Por esta razón, el uso del chol en el contexto escolar observado se debe en gran medida al bilingüismo de los niños, quienes usan el chol en el aula mientras el maestro no está impartiendo la clase o en los espacios recreativos, como las canchas deportivas.

En lo que respecta a los espacios en las iglesias, tanto el chol como el español están presentes en la comunicación, a pesar de que los choles reconocen el fomento del uso de la lengua local por parte de algunos líderes religiosos locales, particularmente en los cultos católico y presbiteriano (entrevista con doña Esther,

\footnotetext{
14 Un caso ilustrativo del proceso de adquisición del chol en el contexto escolar es el que se describe en la nota 7. En este ejemplo el niño de tercer grado no ha aprendido aún a usar el clasificador numeral para referentes humanos, tyikil, y en su lugar usa el clasificador genérico p'ej, lo que resulta en una secuencia inaceptable para el contexto de la pregunta formulada. Para los detalles sobre el uso de los clasificadores numerales en chol, véase Arcos (2009).
} 
17/08/2018). En las celebraciones religiosas de los fines de semana prevalece el chol en las lecturas bíblicas, los sermones y las alabanzas. Hay que destacar que algunos pastores o catequistas usan versiones de la Biblia traducidas al chol y también se cuenta con himnarios en esta lengua maya. Sin embargo, algunos avisos previos o posteriores a las celebraciones religiosas pueden darse en español, dependiendo de la persona que las anuncie. Además, cuando llegan los curas católicos a oficiar conmemoraciones especiales en las comunidades, la misa es en español, aunque los coristas cantan en chol, como sucedió a mediados de agosto de 2018 en Loma Linda, en la zona de Marqués de Comillas, donde el cura de Pico de Oro bautizó a varios niños. La comunicación con el cura, aun después de la ceremonia, aconteció en español, pero la mayoría de los feligreses usó el chol durante la convivencia. Es interesante notar también que al evento religioso fueron invitados músicos de la Unión para que cantaran, y los integrantes de la agrupación musical eran todos adolescentes que se comunicaban entre ellos tanto en español como en zoque.

El último contexto que se analiza en este artículo es el de los ámbitos del comercio y de la atención al turismo nacional y extranjero. Los choles de Frontera Corozal y los de la frontera sur de Marqués de Comillas tienen como principal centro comercial la localidad de Benemérito de las Américas, aunque la propia comunidad de Frontera Corozal y otros poblados como Nuevo Orizaba también juegan un papel importante en el intercambio comercial entre poblaciones de México y Guatemala, que incluyen principalmente productos como maíz, frijol y resina. Los mismos pobladores reconocen que cuando hay abundancia de frijoles en las comunidades choles, venden este producto a compradores guatemaltecos, pero en tiempos de escasez «vuelven a comprar sus propios productos». En todas estas interacciones comerciales, la comunicación transcurre en español.

Es evidente que el bilingüismo chol-español es lo que caracteriza la comunicación de las personas de Frontera Corozal. Es también notorio que en el núcleo familiar se está perdiendo el uso de la lengua local, puesto que el español también está presente como lengua de comunicación en el seno de los hogares, que se consideraban ámbitos tradicionales de uso de la lengua local. Matras (2009:50) destaca que en los estudios sobre la estabilidad o el mantenimiento lingüístico en población migrante, la segunda y tercera generación de las familias lidiarán con la importancia de la lengua mayoritaria en casi todas sus actividades, hasta confinar a la lengua materna al dominio doméstico. En este mismo tenor, Thomason (2015:29-30) sentencia que los niños bilingües, al tomar decisiones 
acerca de sus lenguas, optan siempre por usar solo una de ellas, típicamente la dominante. Estas generaciones que mencionan ambos autores juegan un papel importante en el mantenimiento de la lengua local, con una correlación directa con sus actitudes lingüísticas, tema que se describe brevemente en el apartado que sigue.

\section{Las actitudes lingüísticas de los choles de Frontera Corozal}

Las actitudes lingüísticas son «manifestaciones valorativas que proyectan la percepción que se tiene hacia las personas de grupos diferentes que hablan determinada lengua [...] las actitudes hacia las variedades de una lengua o hacia lenguas distintas son también hacia los hablantes de las mismas, por lo que su estudio no sólo puede contribuir en la explicación de los procesos de variación lingüística, sino también proporcionar información para entender las relaciones interculturales» (Castillo, 2007:26). En el contexto de los cholhablantes de Frontera Corozal encontramos tanto valoraciones sobre las dos variantes dialectales de la lengua local como opiniones con respecto al español. Por un lado, hallamos en esta localidad opiniones sobre el uso predominante de la variante dialectal de Tumbalá, por ser la variedad que cuenta con mayor número de hablantes; aunque en la opinión de los choles que viven fuera del poblado hay una mezcla de las dos variantes dialectales. Por otro lado, al entrevistar a choles que tienen hijos en preescolar o primaria, estos manifestaron el deseo de que los educandos tengan un buen dominio del español para que en el futuro puedan obtener un trabajo bien remunerado en los contextos urbanos. Indudablemente, tales opiniones están contribuyendo a que haya cada vez menos hablantes del chol o a que haya una competencia comunicativa deficiente de los pequeños en esta lengua maya.

Para Appel y Muysken (1996:30), los resultados de los estudios de las actitudes lingüísticas ayudan a comprender la relación entre lengua e identidad. Estos mismos autores destacan el uso de dos metodologías que se han empleado en el estudio de las actitudes lingüísticas, como es la perspectiva conductista y la mentalista. ${ }^{15}$ El empleo de estas metodologías incluye evaluaciones de grabaciones por parte de los hablantes y de la aplicación de cuestionarios sobre el uso de una lengua específica. En general, las valoraciones que se hacen sobre las lenguas o

\footnotetext{
15 Perspectiva conductista (las actitudes se estudian mediante la observación de las respuestas a determinadas lenguas en interacciones reales); perspectiva mentalista (las actitudes son un estado interno y mental, aunque solo pueden ser inferidas por el comportamiento).
} 
sus variedades se han concentrado, por un lado, en la idea de que una variedad es mejor o más atractiva que otra (valor inherente) y, por otro lado, en la creencia de que la variedad que usa el grupo con más prestigio es la mejor o más atractiva (valor impuesto) (Appel y Muysken, 1996:29-30). A pesar de que está pendiente la aplicación sistemática de las metodologías para estudiar las actitudes lingüísticas de los choles, las opiniones obtenidas hasta ahora apuntan a que los hablantes de cada variante dialectal de esta lengua maya consideran que la variedad propia es «la correcta» o «la mejor». Sin embargo, la confluencia de ambas variantes dialectales en Frontera Corozal, por la migración, ha provocado que los pobladores oriundos de Tila adopten algunas formas léxicas y gramaticales del chol de Tumbalá y que estos a su vez simplifiquen el patrón entonacional, asemejándolo a la variedad de Tila. La profesora Hilda explica que el cambio puede observarse desde el contexto escolar cuando un niño es señalado por sus propios compañeros por usar una palabra ajena a la variedad tumbalteca. Una sola experiencia de esta naturaleza es suficiente para que un niño adopte la forma no señalada y la use en los contextos que considere pertinente (entrevista, 21/03/2018). El predominio del uso de una variedad en el contexto escolar no parece ser por razones de prestigio, sino por la cuestión cuantitativa (De la Torre, 1994:84). Esto a su vez explicaría por qué los adultos también hacen acomodaciones lingüísticas en su interacción cotidiana, como es el caso de don José, ejemplificado en (4) y (5), arriba.

Al hablar de las consecuencias lingüísticas del proceso de urbanización, Butragueño destaca la importancia de la evaluación subjetiva del lenguaje, es decir, "las consideraciones que los hablantes hacen acerca de las lenguas y las variedades lingüísticas [...] a partir de prejuicios, actitudes [...], creencias de todo tipo, inseguridades que pueden estar en la base de la aceptación de novedades lingüísticas» (Butragueño, 2010:1051). Este autor subraya la importancia del estudio de las actitudes lingüísticas porque, entre otros aspectos, nos ofrece una explicación de cómo los hablantes eligen entre qué lengua o qué estilo de habla emplear, entre si sumarse o no a la difusión de ciertos hábitos lingüísticos (Butragueño, 2010:1051). Aunque este planteamiento se deriva de una consideración en un contexto urbano, evidentemente aplica para el ámbito rural de Frontera Corozal. En este lugar se puede percibir una valoración positiva del chol tumbalteco, factor que podría estar propiciando las acomodaciones lingüísticas de habitantes originarios del municipio de Tila, como los ejemplos de habla de don José mencionados previamente. 
Respecto al uso del español, los choles de Frontera Corozal lo consideran útil para obtener un empleo bien remunerado y, por lo tanto, una mayor posibilidad de movilidad social. Esto contrasta con el contexto urbano, en el que la evaluación se centra en la riqueza de su vocabulario, por la existencia de una literatura abundante, por ser la lengua oficial de varios países, por su larga historia, por su timbre y musicalidad (Butragueño, 2010:1051). Por esta razón, los padres choles que representan la segunda generación de pobladores en Frontera Corozal prefieren hablarles en español a sus hijos. Esto es lo que ha notado el director de la escuela primaria Jaime Torres Bodet, quien afirma que «algunos niños que llegan a esta escuela primaria lo hacen sin dominar la lengua local, esto porque los padres lo ven como inviable para comunicarse más allá de la localidad» (entrevista, 21/03/2018).

De la Torre notó que en El Lacandón había una amplia base de pobladores jóvenes desligados de su lugar de origen, «tanto niños como niñas han estado expuestos al contacto plurilingüe y en condiciones de mayor cambio sociocultural; ahora, ambos tienen posibilidad de una interacción multilingüe que irá repercutiendo en el futuro de esta población» (De la Torre, 1994:83). La generación que menciona este investigador es la que corresponde ahora a los padres de los niños que se destacan por el uso de la lengua española en las escuelas de nivel básico.

Si tú te vas con primer grado, la mayoría no los escuchas hablar en chol. La mayoría se comunican en español. Porque así viene desde la casa la enseñanza. Tal vez sí lo entienden, pero no lo pueden pronunciar y si lo pronuncian no lo dicen de la manera correcta. Estoy seguro de que sí lo entienden, mas no se pueden expresar como un niño que lo ha venido aprendiendo desde chiquito. Porque se escucha en el sonido cómo lo pronuncian (entrevista, 21/03/2018).

Se puede ver entonces que en Frontera Corozal son complejos los lugares en los que ocurre la práctica cotidiana del chol. En el ámbito educativo, aunque es evidente el uso del español, el chol está también presente en la comunicación de los niños, especialmente durante el tiempo de recreo. Es en este espacio en donde los niños, que no hablan el chol o son hablantes pasivos, lo desarrollan o tienen la oportunidad de practicarlo. Al parecer, el asunto de la elección de una variante dialectal para la interacción comunicativa no ha representado un obstáculo para su práctica. Este hecho contrasta con casos observados en algunos estudios lingüísticos de otras latitudes, como los que reporta Thomason (2015). 
Según esta autora, los jóvenes chinook cambiaron el uso de la lengua local por el inglés cuando los ancianos se burlaban de su forma de hablar porque, al parecer, cometían muchos errores. ${ }^{16}$ Para evitar la burla y teniendo otra lengua que podían usar más fácilmente, los jóvenes simplemente eligieron usar el inglés y dejaron de tratar de emplear el chinook. Este mismo resultado se reporta para algunas variedades del sueco que se introdujo en territorio estadounidense por la migración. La burla hacia los hablantes de dialectos del sueco que no gozaban de cierto prestigio provocó la adopción del inglés. A pesar de que hay opiniones no favorables para la variante del chol de Tila en la localidad de estudio, es evidente que esta actitud no está causando el abandono de la lengua local por parte de las nuevas generaciones. La adopción de elementos lingüísticos ajenos a la variedad propia nos permite vislumbrar un futuro alentador para la vitalidad del chol en Frontera Corozal, debido a que persiste una tendencia opuesta al pronóstico de Thomason (2015:29), «si la comunidad no puede aceptar una versión alterada de su lengua heredada, la lengua estará condenada a la muerte».

\section{Conclusiones}

En este artículo se presentaron algunas características sociolingüísticas del poblado de Frontera Corozal, del municipio de Ocosingo, Chiapas. Lo descrito permite entender la vitalidad de esta lengua maya en un núcleo de población creado hace aproximadamente medio siglo. El estudio es relevante por varias razones. En primer lugar, ofrece elementos que permiten preguntar cómo está aconteciendo la adquisición y la transmisión intergeneracional del chol en este pueblo. Por otra parte, resalta las consecuencias lingüísticas de la confluencia de dos variantes dialectales, plenamente reconocidas por los hablantes. Por último, permite dimensionar el desafío de la lengua chol en el mantenimiento de los ámbitos de uso frente al español, principalmente en la vida escolar, religiosa y familiar.

Lo expuesto en este estudio confirma la idea de que la migración altera la vitalidad lingüística, puesto que hay una tendencia a que la segunda o tercera generación de la población migrante abandone la lengua minoritaria (Matras, 2009). En forma parecida a lo que ocurre con la migración urbana, en la que confluyen lenguas, dialectos o estilos particulares al alcance de los usuarios (Butragueño,

\footnotetext{
${ }^{16}$ La lengua chinook se habla entre los lindes de Washington y Oregón.
} 
2010), los choles migrantes en el contexto rural descrito en este estudio tuvieron un contacto plurilingüe con posibilidad de interactuar en las dos variantes dialectales que convergieron en la colonización. La particularidad de la zona de estudio es que la actitud positiva hacia el chol por parte de los hablantes de la primera generación ha mantenido viva esta lengua maya, a pesar de que el español está ya presente en muchos hogares. Romero (2017) menciona que esta es la constante que se reporta en los estudios sociolingüísticos en poblaciones mayas. Además, los señalamientos que se derivan por el uso de algunas formas propias de una variante dialectal no han sido un factor decisivo en la decadencia en el uso del chol entre las nuevas generaciones, contrario a lo observado en otras latitudes (Thomason, 2015). La vitalidad del chol en este lugar pareciera estar ligada al aislamiento geográfico, a pesar de la constante presencia del turismo (portadores de lenguas dominantes) y de la existencia de programas televisivos nacionales y extranjeros a través de señales satelitales.

Indudablemente, hay algo de valoración positiva de los cholhablantes hacia su lengua materna, lo que ha contribuido a su mantenimiento. Esta actitud está asociada con la generación de una nueva identidad chol que está borrando las diferencias que se enarbolaban en los lugares de origen de los fundadores del pueblo (choles de Tila versus choles de Tumbalá). Las diferencias están claramente identificadas en cada variante dialectal por parte de los hablantes; sin embargo, en este nuevo asentamiento los choles de Tila están ajustando su forma de comunicación al chol de Tumbalá y estos a su vez están cambiando patrones entonacionales y rasgos fonéticos en algunos sonidos para crear una variedad intermedia, en vez de optar por el uso del español. La actitud a la que se hace mención ha facilitado la vitalidad del chol en la comunidad. El hecho de que los choles estén optando por cambiar su forma de habla heredada de su lugar de origen ha provocado que aún no se perciba un desplazamiento dramático del chol en la vida cotidiana.

Entre las tareas pendientes, hace falta aplicar sistemáticamente las metodologías que mencionan Appel y Muysken (1996) para entender más a fondo los factores que contribuyen al mantenimiento del chol en esta zona de El Lacandón y la razón, más allá del aspecto cuantitativo, de la preferencia por la variante de Tumbalá. Habría que ver también si entre los choles que migraron a Kalakmul, Campeche (Rodríguez, 2012), se presentan situaciones lingüísticas similares a las descritas aquí. 
Por otro lado, esta investigación permitió identificar los aspectos fonéticos, gramaticales y léxicos que se destacan en las acomodaciones lingüísticas, ejemplificadas en el habla de algunas personas entrevistadas. Sobre este punto hace falta diseñar una metodología para obtener datos que nos permitan examinar qué tan extendido está este fenómeno de acomodación en la población en general.

Por último, es conveniente conocer a detalle cómo está aconteciendo la socialización infantil y la transmisión intergeneracional del chol en las familias. Esto nos permitiría identificar cuál es la lengua materna de las nuevas generaciones en Frontera Corozal.

\section{Agradecimientos}

Agradezco a Telma Can, Fernando Guerrero, Fausto Bolom y a Danny Law, por los comentarios realizados a versiones preliminares del documento. Agradezco también las recomendaciones puntuales recibidas de parte de los revisores anónimos, mismas que contribuyeron al enriquecimiento del trabajo.

\section{Referencias bibliográficas}

Appel, René y Pieter Muysken, 1996, Bilingüismo y contacto de lenguas, Barcelona, Ariel. Arcos López, Nicolás, 2009, Los clasificadores numerales y las clases nominales en ch'ol, tesis de maestría inédita, México, Centro de Investigaciones y Estudios Superiores en Antropología Social (CIESAS).

Butragueño, Pedro Martín, 2010, «El proceso de urbanización: consecuencias lingüísticas», en Rebeca Barriga Villanueva y Pedro Martín Butragueño (dirs.), Historia Sociolingüística de México, vol. 2, México, El Colegio de México, pp. 997-1093.

Castellanos Navarrete, Antonio, 2018, «Palma de aceite en tierras campesinas: la política de las transformaciones territoriales en Chiapas, México», Revista Pueblos y Fronteras digital, 13, pp. 1-34.

Castillo Hernández, Mario Alberto, 2007, Mismo mexicano pero diferente idioma: identidades y actitudes lingüísticas en los maseualmej de Cuetzalan, México, Instituto Nacional de Antropología e Historia/IIA-UNAM.

Cruz Pérez, Manuel de Jesús, 2014, Mitos, cosmovisiones e historias choles en Sabanilla, Chiapas, tesis de maestría inédita, México, CIESAS.

De la Torre Yarza, Rodrigo, 1994, Chiapas: entre la Torre de Babel y la lengua nacional, México, CIESAS. 
Field, Fredric W., 2002, Linguistic borrowing in bilingual contexts, Ámsterdam/ Philadelphia, John Benjamins Publishing Company.

González Ponciano, Jorge Ramón, 2004, «Marqués de Comillas: cultura y sociedad en la selva fronteriza México-Guatemala», en Juan Pedro Viqueira y Mario Humberto Ruz (eds.), Chiapas, los rumbos de otra historia, 2ª . ed., México, UNAM/CIESAS, pp. 425-444.

Instituto Nacional de Lenguas Indígenas (INALI), 2015a, Indicadores básicos sobre la agrupación ch'ol, en <https://site.inali.gob.mx/Micrositios/estadistica_basica/estadisticas2015/pdf/agrupaciones/chol.pdf> (consulta: 20/05/2019).

INALI, 2015b, Indicadores básicos sobre la agrupación tseltal, en <https://site.inali.gob. mx/Micrositios/estadistica_basica/estadisticas2015/pdf/agrupaciones/tseltal.pdf> (consulta: 20/05/2019).

INALI, 2015c, Indicadores básicos sobre la agrupación tsotsil, en <https://site.inali.gob. mx/Micrositios/estadistica_basica/estadisticas2015/pdf/agrupaciones/tsotsil.pdf > (consulta: 20/05/2019).

Instituto Nacional de Pueblos Indígenas (INPI), 2018, Atlas de los Pueblos Indígenas de México, en <http://atlas.cdi.gob.mx> (consulta: 20/05/2019).

Law, Danny, 2017, «Language contacts with(in) Mayan», en Judith Aissen, Nora England y Roberto Zavala (eds.), The Mayan languages, Nueva York, Routledge, pp. 112-127.

López López, Rubén, 2005, Una aproximación al léxico comparativo del chol de los municipios de Sabanilla, Tila y Tumbalá, tesis de maestría inédita, México, CIESAS.

Matras, Yaron, 2009, Language contact, Cambridge, Cambridge University Press.

Robertson, John S., Danny Law y Robbie A. Haertel, 2010, Colonial Ch'olti': The Seventeenth-Century Morán Manuscript, Oklahoma, University of Oklahoma Press.

Rodríguez Ceja, Gabriela Eugenia, 2012, Enfermar y sanar. Persona, cuerpo social y cosmos en la vida cotidiana chol en Calakmul, tesis doctoral inédita, México, UNAM.

Romero, Sergio, 2017, "The labyrinth of diversity. The Sociolinguistics of Mayan Languages», en Judith Aissen, Nora England y Roberto Zavala (eds.), The Mayan languages, Nueva York, Routledge, pp. 379-400.

Sánchez-Marco, Francisco, 1976, Acercamiento histórico a la sociolingüística, México, Secretaría de Educación Pública/INAH.

Thomason, Sarah G., 2015, Endangered languages, An introduction, Cambridge, Cambridge University Press.

Vázquez Álvarez, Juan Jesús, 2002, Morfología del verbo de la lengua chol de Tila, Chiapas, tesis de maestría inédita, México, CIESAS.

Vázquez Álvarez, Juan Jesús, 2011, A Grammar of Chol, a Mayan Language, tesis doctoral inédita, Austin, Universidad de Texas.

Vos, Jan de, 1980, La paz de Dios y del Rey. La conquista de la Selva Lacandona (15251821), México, Fondo de Cultura Económica.

Vos, Jan de, 1994, Oro verde, la conquista de la Selva Lacandona por los madereros tabasqueños, 1882-1949, México, FCE/Instituto de Cultura de Tabasco.

Vos, Jan de, 2002, Una tierra para sembrar sueños. Historia reciente de la Selva Lacandona, 1950-2000, México, FCE/CIESAS. 
Vos, Jan de, 2004, «El Lacandón: una introducción histórica», en Juan Pedro Viqueira y Mario Humberto Ruz (eds.), Chiapas, los rumbos de otra historia, 2a. ed., México, UNAM/CIESAS, pp. 331-361.

Wardhaugh, Ronald, 1998, An introduction to Sociolinguistics, Oxford, Blackwell Publishers.

Zavala, Roberto, 1992, El kanjobal de San Miguel Acatán, México, UNAM. 